\title{
Mucus transportability: the bovine trachea and frog palate models compared
}

\author{
P.J. Wills, K. Pritchard, P.J. Cole
}

Mucus transportability: the bovine trachea and frog palate models compared. P.J. Wills, K. Pritchard, P.J. Cole. (OERS Journals Ltd 1998.

ABSTRACT: For nearly $30 \mathrm{yrs}$, the mucus-depleted frog palate has been used to measure the ciliary transportability of respiratory and other mucus gels, but the data obtained from this amphibian digestive system may not be applicable to human airway mucociliary clearance. This study compared this model with the mucus-depleted bovine trachea, a mammalian respiratory system. Assessments were made of the reproducibility of each model, and of the behaviour of sputum subjected to changes to its salinity or hydration.

The bovine tracheal model was more reproducible than the frog palate. On the trachea but not the frog palate, sputum was transported more slowly than mucus from healthy animals. Increasing the salinity of sputum caused it to be transported $129 \%$ more quickly by the trachea $(p=0.001)$, but made no significant change to its transportability by the frog palate. Removal of water by evaporation led to an $83 \%$ increase in its bovine tracheal transportability but a $60 \%$ fall in its frog palate transportability $(p<0.001)$.

Therefore, the models make opposite predictions for the clinical value of altering mucus osmolality. The applicability of the frog palate model in the study of airway mucociliary clearance should be seriously questioned.

Eur Respir J 1998; 12: 837-841.
Host Defence Unit, Imperial College at Royal Brompton National Heart and Lung Institute, London, UK.

Correspondence: P.J. Wills, Host Defence Unit, Imperial College at Royal Brompton, National Heart and Lung Institute, Emmanuel Kaye Building, Manresa Road, London SW3 6LR, UK

Fax: 441713518338

Keywords: Bovine, frog, mucociliary clearance, osmolarity, sputum

Received: August 251997

Accepted after revision May 191998

P.J.W. received a grant from Bayer Research.
The mammalian airway surface forms a major interface between the individual and the environment. The mucociliary escalator is an important defence against unwanted airborne substances. Mucus with entrapped material is conveyed by the cilia towards the pharynx, where it is normally eliminated by swallowing.

In conditions characterized by sputum production, such as acute respiratory infections, chronic bronchitis and bronchiectasis, including cystic fibrosis $(\mathrm{CF})$, mucociliary clearance is delayed [1]. The abnormally prolonged retention of infected mucus in the airways is arguably a major cause of the chronic inflammatory damage seen in these chronic conditions [2].

Efficient mucociliary clearance requires that both the cilia and the mucus function optimally. Ciliary damage accounts in part for the mucus retention in these diseases [2], but the continuing efforts to discover effective mucoactive drugs testify to the hope that it may be possible to alter the mucus so that it is expelled more easily.

The ability of mucus to be cleared efficiently by cilia can be measured ex vivo using explants of ciliated tissue depleted of endogenous mucus. Traditionally, the frog palate has been used for this purpose in numerous studies of respiratory mucus and other gels [3]. The authors have recently developed a model with the same scope, but using the bovine trachea. With this system two interesting observations were made that had not been reported with the frog palate model. Firstly, sputum from patients with bronchiectasis, $\mathrm{CF}$ and chronic bronchitis was transported more slowly than mucus obtained from healthy human and animal lungs $[4,5]$. Secondly, increasing the hydra- tion of sputum markedly impaired its transportability, whereas increasing its osmolality by adding sodium chloride or removing water by evaporation caused its ciliary transportability to increase [6]. These observations may have particular relevance in the pathogenesis of $\mathrm{CF}$, because in this illness an epithelial defect in sodium and chloride transport results in life-shortening chronic bronchial infection [7].

It might be expected that if the bovine trachea model yielded results different from the frog palate, it would better reflect the properties of respiratory mucus that lead to its efficient expulsion from the human lung. It is a mammalian respiratory system, faced with the same task as the human airway. In contrast, the frog palate is an amphibian digestive system, which functions mainly to trap and help to ingest flies and other particles of food. Mucociliary transport in the frog palate is in a caudal direction, whereas it is cranial in the mammalian trachea. Therefore, a comparison of these models was undertaken, examining the transportability of samples of sputum and healthy lung mucus on both systems. An investigation was made of the effect on ciliary transportability of inspissating sputum by evaporation of water and of directly altering its salinity. The effect of altered salinity on the beat frequency of frog palate cilia was measured, as it had been previously for bovine tracheal cilia [6].

\section{Methods}

Sputum samples (purulent or mucopurulent) were collected from patients with $\mathrm{CF}$ or non-CF bronchiectasis, 
placed on ice immediately and frozen within $6 \mathrm{~h}$. Samples with visible amounts of saliva were not used. Addition to sputum of $10 \%$ of its volume of saliva was easily detectable macroscopically, so salivary contamination of the samples was considerably less than this in all cases. Bovine tracheal mucus or frog palate mucus was collected from the cut end of the preparations, where it collected during the depletion process.

The transportability of sputum was measured at $37^{\circ} \mathrm{C}$ on the mucus-depleted bovine trachea as described previously [4]. In brief, explants of bovine trachea $(8 \mathrm{~cm} \times 3$ $\mathrm{cm})$ were depleted of endogenous mucus by prolonged incubation exposed to the air but kept moist with phosphate-buffered saline (PBS), followed by repeated application of $0.5 \mathrm{~mL}$ of bovine tracheal mucus. The trachea was deemed to be depleted of mucus when it no longer transported small metal particles. The transport rates of samples of sputum or bovine tracheal mucus were then measured by placing approximately $50 \mu \mathrm{L}$ at the distal end and recording the distance travelled at $15 \mathrm{~s}$ intervals using the naked eye. The transport rate was deduced from at least four data points.

The frog palate system was used as described previously [3]. Palates of Rana pipiens were excised, washed in frog Ringer solution and placed in a humid box. Mucus depletion was accomplished by overnight incubation at $4^{\circ} \mathrm{C}$ followed by approximately $1 \mathrm{~h}$ at $25^{\circ} \mathrm{C}$. Mucus transport was observed with a microscope, and its rate was calculated from the time taken for a sample of mucus (approximately $5 \mu \mathrm{L}$ ) to travel $0.5 \mathrm{~cm}$. Measurements were performed at least in triplicate and the mean was calculated.

For both the frog palate and the bovine tracheal assays, the transportability of a sputum sample was expressed as an index. This was its rate of movement expressed as a percentage of that of endogenous frog or bovine mucus which had been collected during the depletion process. For example, a sample with a transportability index of 25 was transported at a quarter of the rate of endogenous frog or bovine mucus.

The within-day and between-day variability of the sputum transportability measurements were assessed using the frog palate system, as had previously been done with the bovine trachea [4]. A sample of bronchiectasis sputum was divided into six aliquots and frozen at $-20^{\circ} \mathrm{C}$. One aliquot was thawed on each of 6 days, kept on ice and assayed six times each day.

Three samples of bovine tracheal mucus were each assayed seven times on the frog palate, each sample on a different day. The salinity of the sputum was altered either by simply adding sodium chloride or by incubating in an excess of saline solution. For the first method, sputum samples from 14 patients with $\mathrm{CF}$ were divided into two aliquots of approximately $0.5 \mathrm{~mL}$ and solid sodium chloride was added to a final concentration of $0.5 \% \mathrm{w} / \mathrm{w}$; the control aliquot was untreated. After incubating overnight at $4^{\circ} \mathrm{C}$, which allows full diffusion of the salt, the transportabilities were measured. In this way, the salinated sputum differs from the control only in having extra sodium chloride. In the second protocol, approximately $1 \mathrm{~mL}$ of sputum was incubated at $4^{\circ} \mathrm{C}$ for $48 \mathrm{~h}$ in excess $(20 \mathrm{~mL})$ PBS (Dulbecco A (Oxoid Ltd., Basingstoke, UK), with peni-cillin 50 units $\cdot \mathrm{mL}^{-1}$, streptomycin $50 \mu \mathrm{g} \cdot \mathrm{mL}^{-1}$ and phenylmethylsulphonylfluoride $0.1 \mathrm{mM}$ ) in tonicities ranging from 0-600 mosmol. $\mathrm{L}^{-1}$, and then removed with forceps. Isotonic PBS contained $137 \mathrm{mM}$ sodium chloride, $3 \mathrm{mM}$ potassium chloride, $8 \mathrm{mM}$ disodium hydrogen phosphate and $1.5 \mathrm{mM}$ potassium dihydrogen phosphate, $\mathrm{pH}$ 7.3. PBS of different tonicities contained the same proportion of solutes. Two CF and two non-CF samples were used. Here, the sputum solute composition will be nearly re-placed by that of the incubating solution, and it is possible to lower as well as to raise the salinity of the gel.

The osmolality of sputum was also increased by inspissation. Sputum samples from 16 patients (eight CF and eight non-CF bronchiectasis) were each divided into two aliquots of $0.5-1.5 \mathrm{~g}$. One aliquot was placed in a shallow dish under a current of air for 2-3 h with occasional gentle stirring until $48-52 \%$ of its original weight was lost; the other aliquot was kept for the same time at ambient temperature in a sealed container.

The ciliary beat frequency of the epithelium was measured photometrically as described previously [8]. Cilia were viewed with transmitted light at a magnification of 320 and a Leitz MPV compact microscope photometer (Wetzler, Germany) transduced the light intensity into an electrical signal. The epithelium from an explant of bovine trachea or a frog palate was dissected into $2-5 \mathrm{~mm}$ pieces, then kept in isotonic PBS or frog Ringer solution as appropriate. Samples were then placed in a transparent dish containing PBS of different osmolarities for $15 \mathrm{~min}$ at $37^{\circ} \mathrm{C}$ or $25^{\circ} \mathrm{C}$, respectively, and the ciliary beat frequency was measured. For each saline concentration, measurements of the ciliary beat frequency were made from 10 different areas and the mean was calculated.

Nonparametric tests for significance were used throughout, with the Minitab® statistical program (PA, USA).

\section{Results}

\section{Reproducibility measurements}

The mean transportability index of the sputum sample assayed 36 times on the frog palate was 48 (range 9-138, coefficient of variation 0.75 , SD 36.4 , SEM 6.1 ). This is approximately three times the variability obtained in a sample from the same patient with the bovine trachea in a similar study [4]. The within-day coefficients of variation were between 0.15 and 0.34 , compared with between 0.08 and 0.28 with the bovine trachea.

\section{Transportability of sputum and bovine tracheal mисus}

The transportability indices (mean \pm SEM) of all 30 sputa (from 22 patients with $\mathrm{CF}$ and eight with non-CF bronchiectasis) were $26 \pm 2.4$ on the bovine trachea and $80 \pm 9.8$ on the frog palate $(\mathrm{p}=0.001$, Wilcoxon test). For the $22 \mathrm{CF}$ sputa the bovine and frog transportability indices were $29 \pm 2.6$ and $74 \pm 10.7$ ( $p=0.001$, Wilcoxon test) and for the eight non-CF bronchiectasis sputa the respective transportability indices were $17 \pm 4.8$ and $98 \pm 22.2(p=0.014)$.

The three samples of bovine tracheal mucus had transportability indices (mean \pm SEM for seven measurements) on the frog palate of $40 \pm 5.0,29 \pm 5.3$ and $114 \pm 6.5$. The over-all mean transportability index of bovine tracheal mucus on 
Table 1. - Transportability indices of 14 samples of cystic fibrosis sputum with or without the addition of sodium chloride, to $0.5 \%$

\begin{tabular}{lcc}
\hline & \multicolumn{2}{c}{ Transportability index } \\
\cline { 2 - 3 } & Bovine trachea & Frog palate \\
\hline Untreated & $28 \pm 4$ & $66 \pm 15$ \\
Added $\mathrm{NaCl}$ & $64 \pm 5$ & $46 \pm 14$ \\
Change $\%$ & +129 & -30 \\
p-value & 0.001 & 0.68
\end{tabular}

Data are mean \pm sem. Probabilities were calculated using the Wilcoxon test.

the frog palate was 61 . This was not significantly different from the transportability of sputum on the frog palate ( $\mathrm{p}>0.13$, Mann-Whitney U-test).

\section{Addition of sodium chloride to sputum}

The bovine tracheal and frog palate transportabilities of sputa from 14 patients with $\mathrm{CF}$, untreated and with added salt, are shown in table 1 . On the bovine trachea there was a $129 \%(\mathrm{p}<0.001)$ increase in transportability after adding sodium chloride, as reported previously [6]. On the frog palate, increasing the salinity of the sputum caused a $30 \%$ (nonsignificant) fall in transportability.
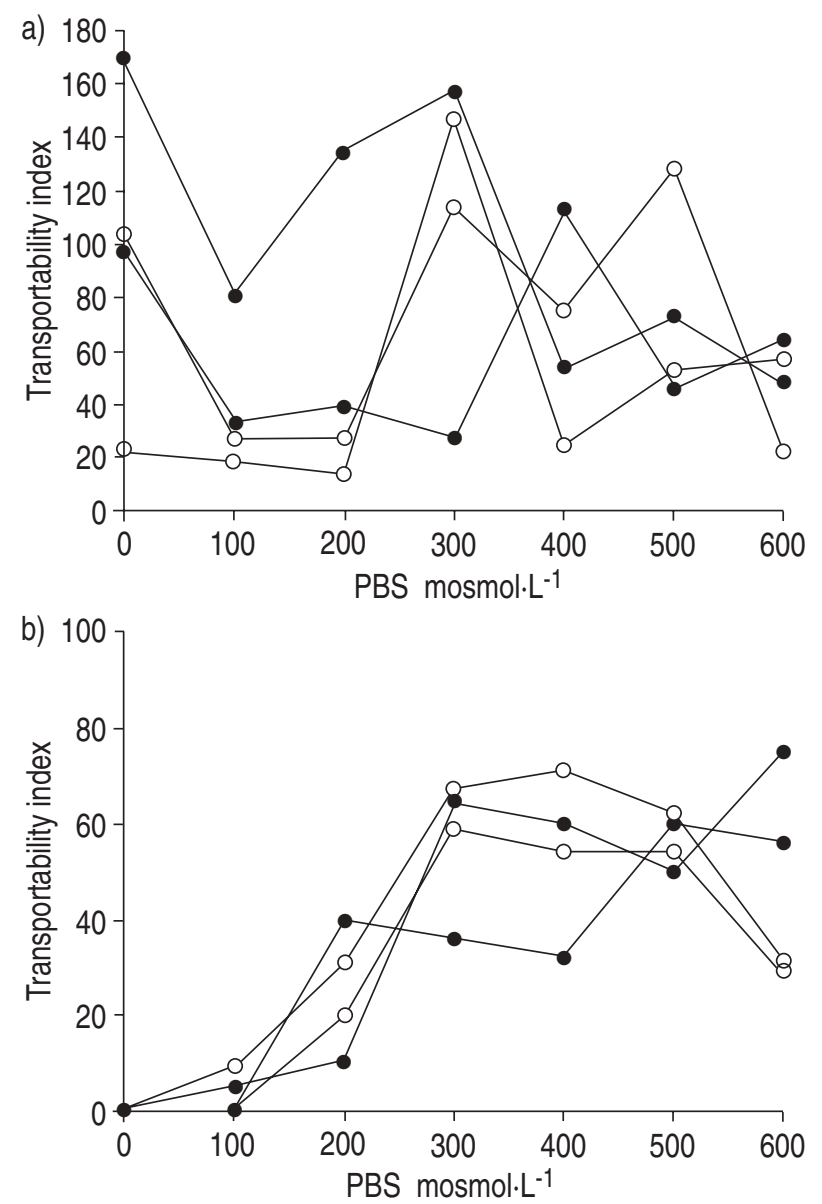

Fig. 1. - a) Frog palate and b) bovine tracheal transportabilities of two cystic fibrosis $(\mathrm{CF})(\bullet)$ and two non-CF sputa $(O)$ after incubation in excess phosphate buffered saline (PBS)
Table 2. - Bovine trachea and frog palate transportabilities of 16 sputa before and after inspissation

\begin{tabular}{lccccccc}
\hline & \multicolumn{4}{c}{ Transportability index } \\
\cline { 2 - 4 } & \multicolumn{3}{c}{ Bovine trachea } & & \multicolumn{3}{c}{ Frog palate } \\
\cline { 2 - 4 } \cline { 6 - 8 } & All & CF & non-CF & All & CF & non-CF \\
\hline Untreated & $24 \pm 3$ & $30 \pm 3$ & $17 \pm 5$ & $92 \pm 13$ & $87 \pm 14$ & $98 \pm 22$ \\
Inspissated & $44 \pm 6$ & $50 \pm 8$ & $38 \pm 8$ & $37 \pm 7$ & $35 \pm 7$ & $38 \pm 14$ \\
Change \% & +83 & +67 & +124 & & -60 & -60 & -61 \\
p-value & 0.001 & 0.035 & 0.014 & $<0.001$ & $<0.014$ & 0.014 \\
\hline
\end{tabular}

Data are mean \pm SEM of eight cystic fibrosis $(\mathrm{CF})$ and eight non$\mathrm{CF}$ subjects. Probabilities were calculated using the Wilcoxon test.

\section{Incubation of sputum in saline solution}

Figure 1 shows the transportabilities of samples of $\mathrm{CF}$ and bronchiectasis sputum after $48 \mathrm{~h}$ incubation in an excess of sodium chloride solution. On the bovine trachea a clear saline dependence is apparent, with optimum transportability occurring when the sputum was incubated in 300-500 mosmol. $\mathrm{L}^{-1}$ saline, as reported previously [6]. Sputa incubated in $<100 \mathrm{mosmol} \cdot \mathrm{L}^{-1}$ saline were virtually untransportable on the bovine trachea. With the frog palate model the transportability showed no obvious saline dependence and sputa incubated in water were transported well.

\section{Inspissation of sputum}

The mean transportability indices of 16 sputa (eight from $\mathrm{CF}$ and eight from non-CF bronchiectasis patients) before and after inspissation are shown in table 2. Water loss caused an $83 \%$ increase in transportability on the bovine trachea, but a $60 \%$ fall in transportability on the frog palate; both changes were highly significant.

\section{Ciliary beat frequency}

The beat frequency of the frog palate cilia as a function of salinity is shown in figure 2 . In contrast to bovine tracheal cilia, unstimulated frog palate cilia were often stationary.

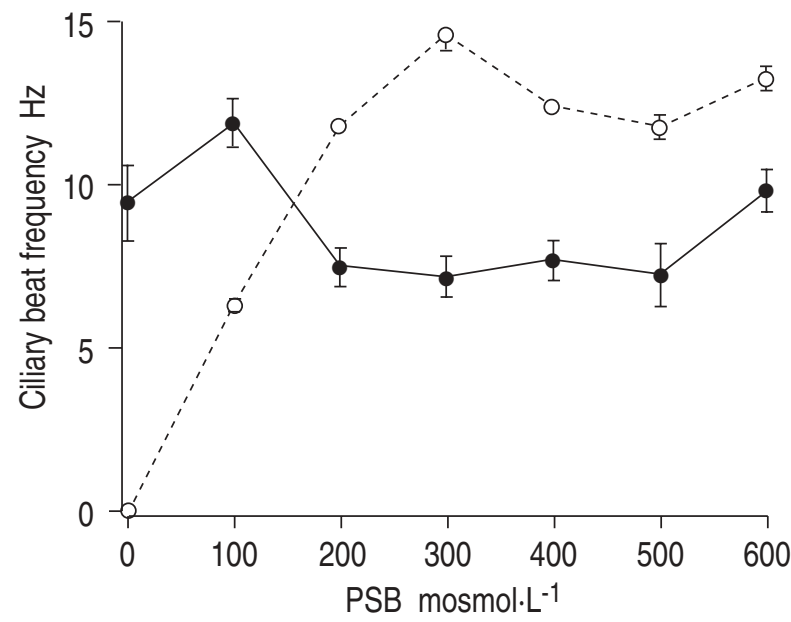

Fig. 2. - Beat frequency of frog palate (•) and bovine tracheal (O) cilia as a function of the salinity of the surrounding liquid. PBS: phosphatebuffered saline. 
Readings of ciliary beat frequencies were therefore made immediately after a mechanical stimulus. With the frog palate cilia, the ciliary beating frequency showed no clear saline dependence and near-optimal beating occurred in distilled water.

\section{Discussion}

The frog palate has been used in studies of ciliary activity since 1948 [9] and in studies of pulmonary mucus for nearly three decades $[10,11]$. The mucus-depleted bovine trachea, a model with the same scope has recently been described and in this work the performance of the two systems in assaying pulmonary mucus was compared. The differences in the results obtained with the two models could have important therapeutic implications.

Firstly, reproducibility was better using the bovine trachea than the frog palate. When multiple aliquots of the same sputum sample were assayed, both the within-day and between-day agreement was much closer with the bovine trachea model. The maximum and minimum values of the transportability index of a sputum sample measured 36 times on the frog palate were 9 and 138, while on the bovine trachea the respective values were 11 and 31 [4].

This study is the first, to the authors' knowledge, that has specifically addressed the question of the reproducibility of the frog palate model. In a previous study [3] up to eight measurements were occasionally required to obtain three within $10 \%$ of each other. The poor reproducibility of the system is probably due mainly to its small size. Because of this, the transport rate of a mucus sample is deduced from the time taken to travel between two points only. With the much longer bovine trachea, time is allowed after application of the mucus sample for a steady state speed to be reached, then the distance travelled at several time points can be measured. A sputum sample often moved more quickly in the first $30 \mathrm{~s}$ or so, before slowing to a steady speed, possibly owing to an interaction with the surface liquid. The smaller size of the sample applied to the frog palate, another constraint imposed by its small size, probably also contributes to its poorer reproducibility, because of the greater and more variable ratio of surface liquid to mucus. The effect of salinity on sputum transportability using the bovine trachea is observed even if the mucus is dipped in the solution for $<30 \mathrm{~s}$. Therefore, the solutes in the very small quantities of mucus applied to the frog palate would be expected to equilibrate rapidly with the liquid on the surface of the palate. In contrast, the tonicity of the larger quantities of mucus that can be placed on the bovine trachea would not be expected to approach that of the surface liquid. In addition, the smaller the sample size, the greater the risk that lack of sample homogeneity could affect the result.

Secondly, the frog is a poikilothermic amphibian and assays of sputum transportability are made at $25^{\circ} \mathrm{C}$, whereas the bovine trachea mucus transport measurements were carried out at $37^{\circ} \mathrm{C}$. It is possible that the rheology of airway mucus and, therefore, its transportability is temperature dependent, although the authors are unaware of data on this matter.

Thirdly, the microscopic behaviour of the cilia was different in the two systems. Bovine tracheal cilia, like human respiratory cilia, beat at a nearly constant rate, but the frog cilia were often stationary and could be induced to beat with a physical stimulus. It is not clear whether this results in any difference in mucus transportability, but it shows that frog and bovine cilia function differently despite their ultrastructural similarities.

Fourthly, the salt dependence of the ciliary beat frequency contrasted markedly. Frog palate cilia function nearoptimally in pure water, whereas bovine tracheal cilia did so at an ionic strength of 300-600 mosmol. $\mathrm{L}^{-1}$. Tonicities of $<100 \mathrm{mosmol} \cdot \mathrm{L}^{-1}$ caused complete stasis of bovine tracheal cilia [6]. Presumably, this reflects the contrasting demands placed on these different epithelia in vivo. The frog palate serves a mainly digestive purpose and, like the human palate, must be able to tolerate exposure to fresh water. The mammalian trachea is never normally required to withstand pure water, being exposed only to airway secretions. Moreover, the tonicity of frog Ringer solution is only approximately two thirds that of mammalian. The poor transportability on the bovine trachea of sputum incubated in very hypotonic solutions is probably due, in part at least, to inhibition of ciliary beating.

Finally, the ciliary transportability of respiratory mucus was different in the two models. On the frog palate, the mean transportability index of the 30 samples (eight from $\mathrm{CF}$ and 22 from non-CF bronchiectasis patients) assayed in this study was three times that on the bovine trachea. Analysing the $\mathrm{CF}$ and non-CF sputa separately yielded similar conclusions, although there was a tendency for the CF sputa to be transported more quickly than non-CF sputa on the bovine trachea. The contrasting behaviour of mucus from healthy animal lungs, which was transported rapidly by the bovine trachea, and sputum, which was transported poorly, was not seen with the frog palate model. The marked increase in sputum ciliary transportability observed on the bovine trachea when the salinity was increased was also not apparent on the frog model. On the contrary, there was a trend in the reverse direction, with reduced frog palate transportability of the sputa with added sodium chloride.

The most striking difference in transportability behaviour was seen with inspissated sputum. Samples of sputum were subjected to a large evaporative water loss, so that the original weight was halved. This procedure resulted in a much thicker and stickier gel, which was transported $83 \%$ more quickly than untreated sputum on the bovine trachea, but $60 \%$ more slowly on the frog palate.

Increasing the hydration of sputum by incubating it in water also leads to opposite changes in transportability in the two systems. Sputum incubated in water is rendered virtually untransportable on the bovine trachea, as shown in this study and elsewhere [6], whereas its transportability on the frog palate is increased [12].

It is possible that the greater water permeability of the bovine trachea allows changes to take place in the composition and rheology of that part of the sputum gel in close contact with the cilia, which may not occur when the sputum is placed on the less permeable frog palate. The bovine tracheal model may therefore be measuring the transportability of a sputum gel which has been modified by an interaction with liquid that has been drawn out of the tracheal epithelium in response to an osmotic stimulus. Rapid shifts of liquid can occur across mammalian airways in vivo, as illustrated by the prompt disappearance from the lungs of inhaled liquid in cases of near drowning. 
Therefore, the two models make different predictions for the value of therapeutic interventions. Using the frog palate system, one would be tempted to conclude that adding water to respiratory mucus makes it more transportable. In contrast, the bovine model makes the possibly counterintuitive prediction that increasing the salinity or reducing the hydration of mucus would aid its ciliary clearance and that adding water or hypotonic solutions would be detrimental. Which model gives greater insight into the pathophysiology of lung mucus retention? How have these contrasting clinical predictions been fulfilled in practice?

Clinical studies of the effect of inhaled therapy suggest that inhalation of water [13] and isotonic saline [14] has little or no effect on mucociliary clearance. One study on the use of nebulized water in bronchiectasis, as an adjunct to postural drainage, suggested that clearance was improved, but no attempt was made to distinguish ciliary from cough clearance [15]. Water inhalation, like hypertonic saline, is an irritant and could increase clearance by provoking cough.

Long-term nocturnal mist tent therapy, once common practice in many countries for the treatment of $\mathrm{CF}$, has long been abandoned. In contrast, hypertonic saline inhalations improve mucociliary clearance in CF [16], chronic bronchitis [17], asthmatics and normal individuals [16]. Other therapies which would be expected to increase the salinity of airway surface liquid have been shown to accelerate lung mucociliary clearance. Nebulized amiloride increases the salinity of CF sputum without altering its hydration [18] and in one study improved in vivo mucociliary (and cough) clearance [14]. Nebulized uridine 5'-triphosphate, a chloride secretagogue, improves tracheobronchial clearance in CF patients [19] and normal individuals [20].

The bovine tracheal model gives an insight into how such therapies may work: they may simply increase the salinity of the retained mucus, in particular the layer of mucus in contact with the cilia. Such a change has a prokinetic effect if the sputum is tested on mammalian respiratory cilia, but not if the sputum is tested on frog palate cilia. Increasing the salinity of sputum lowers its viscosity and elasticity and the rheological change probably accounts for the improved transportability [6].

The use of a mammalian respiratory system for measuring the ciliary transportability of airway mucus has obvious intuitive appeal. The mucus-depleted bovine trachea model, in contrast to the frog palate model, has yielded results with considerable biological plausibility and appears to have power to predict a clinical response, at least as far as the effect of salt is concerned. Respiratory mucus retention is a common and often serious problem; it is suggested that this model has given an insight into its pathophysiology and indicates logical therapeutic approaches.

Acknowledgements: P.C. Turner \& Co. supplied
the bovine tracheas. The authors thank D. Corbey
for technical assistance with the frog palate system.

\section{References}

1. Kobayashi K, Wanner A. Mucociliary clearance and ciliary activity. In: Chung KF, Barnes PJ, eds. Pharmacology of the Respiratory Tract: Experimental and Clinical Research. New York, Dekker, 1995; pp. 621-653.
2. Cole P, Wilson R. Host-microbial interrelationships in respiratory infection. Chest 1989; 95: 217S-221S.

3. Rubin BK, Ramirez O, King M. Mucus-depleted frog palate as a model for the study of mucociliary clearance. J Appl Physiol 1990; 69: 424-429.

4. Wills PJ, Garcia Suarez MJ, Rutman A, Wilson R, Cole PJ. The ciliary transportability of sputum is slow on the mucus-depleted bovine trachea. Am J Respir Crit Care Med 1995; 151: 1255-1258.

5. Wills PJ, Wodehouse T, Abdallah S, Wilson R, Cole PJ. Sputum from bronchiectasis, chronic bronchitis and cystic fibrosis is poorly transported by cilia. Am J Respir Crit Care Med 1995; 151: A244.

6. Wills PJ, Hall RL, Chan WM, Cole PJ. Sodium chloride increases the ciliary transportability of cystic fibrosis and bronchiectasis sputum on the mucus-depleted bovine trachea. J Clin Invest 1997; 99: 9-13.

7. Boucher RC. Human airway ion transport. Part two. Am J Respir Crit Care Med 1994; 150: 581-593.

8. Rutland J, Cole PJ. Non-invasive sampling of nasal cilia for measurement of beat frequency and study of ultrastructure. Lancet 1980; ii: 564-565.

9. Stewart WC. Weight-carrying capacity and excitability of excised ciliated epithelium. Am J Physiol 1948; 152: 1-5.

10. Sadé J, Eliezer N, Silberberg A, Nevo AC. The role of mucus in transport by cilia. Am Rev Respir Dis 1970: 102: 48-52.

11. Puchelle E, Zahm JM, Girard F, et al. Mucociliary transport in vivo and in vitro. Relations to sputum properties in chronic bronchitis. Eur J Respir Dis 1980; 61: 254-264.

12. Girod de Bentzmann S, Pierrot D, Fuchey C, Zahm JM, Morancais JL, Puchelle E. Distearoyl phosphatidylglycerol liposomes improve surface and transport properties of CF mucus. Eur Respir J 1993; 6: 1156-1161.

13. Parks CR, Woodrum DE, Graham CB, Cheney FW, Hodson WA. Effect of water nebulisation on normal canine pulmonary mucociliary clearance. Am Rev Respir Dis 1971; 104: 99-106.

14. App EM, King M, Helfesrieder R, Köhler D, Matthys H. Acute and long-term amiloride inhalation in cystic fibrosis lung disease. A rational approach to cystic fibrosis therapy. Am Rev Respir Dis 1990; 141: 605-612 .

15. Conway JH, Fleming JS, Perring S, Holgate ST. Humidification as an adjunct to chest physiotherapy in aiding tracheo-bronchial clearance in patients with bronchiectasis. Respir Med 1992; 86: 109-114.

16. Robinson M, Regnis JA, Bailey DL, King M, Bautovich GJ, Bye PTP. Effect of hypertonic saline, amiloride and cough on mucociliary clearance in patients with cystic fibrosis. Am J Respir Crit Care Med 1996; 153: 1503-1509.

17. Pavia D, Thomson ML, Clarke SW. Enhanced clearance of secretions from the human lung after the administration of hypertonic saline aerosol. Am Rev Respir Dis 1978; 117: 199-203.

18. Tomkiewicz RP, App EM, Zayas JG, et al. Amiloride inhalation therapy in cystic fibrosis. Influence on ion content, hydration, and rheology of sputum. Am Rev Respir Dis 1993; 148: 1002-1007.

19. Bennett WD, Olivier KN, Zeman KL, Hohneker KW, Boucher RC, Knowles MR. Effect of uridine 5 'triphosphate plus amiloride on mucociliary clearance in adult cystic fibrosis. Am J Respir Crit Care Med 1996; 153: 1796-1801.

20. Olivier KN, Bennett W, Hohneker K, et al. Acute safety and effects on mucociliary clearance of aerosolised uridine 5 'triphosphate +-amiloride in normal human adults. Am J Respir Crit Care Med 1996; 154: 217-223. 